\title{
Glycoside- and acyclic nucleoside-based 6-cyclohexyl-4-aryl-2-oxonicotinonitrile: synthesis and antimicrobial evaluation
}

\author{
Abdalla E. Hassan ${ }^{1,2}$ (D) Reham A. I. Abou-elkhair ${ }^{1,2} \cdot$ Samar M. Mohamed $^{2} \cdot$ Ahmed H. Moustafa $^{2} \cdot$ Reda M. Fikry $^{2} \cdot$ \\ Fathy A. Yassen ${ }^{2}$
}

Received: 6 October 2018 / Accepted: 2 April 2019 / Published online: 9 April 2019

(c) Iranian Chemical Society 2019

\begin{abstract}
Antimicrobial resistance is a global public health threat that signals the identification of new antimicrobial agents with different modes of action. The synthesis and the antimicrobial evaluations of a series of 2-oxonicotinonitriles (2-ONNs) derivatives are described. The parent 2-ONN derivative 3 was synthesized by one-pot four-component reaction: acetyl cyclohexane, $p$-anisaldehyde, ethyl cyanoacetate, and $\mathrm{NH}_{4} \mathrm{OAc}$, in high yield. Density functional theory geometrical equilibrium of the tautomeric forms of $\mathbf{3}$ concluded the predominance of the keto tautomer. Base-catalyzed coupling of $\mathbf{3}$ with several organohalides/alkylating agents provided branching and solubilizing groups to the 2-ONN derivative 3. Alkylated ONNs and free acyclic nucleoside mimics/glycosides derived from the 2-ONN derivative 3 were tested for their antibacterial and antifungal activities. The acyclic nucleoside mimic 13 and the $N^{1}$-propargyl derivative $\mathbf{1 6}$ and $N^{1}$-allyl derivative 18 showed significant activities against $S$. aureus, and $M$. luteus; meanwhile, significant anti-E. coli activity was observed with compounds $\mathbf{3}$ and 18. On the other hand, a significant antifungal activity was observed with $\mathbf{3}$ and with its $N^{1}$-glucoside derivatives $\mathbf{4}$ and $\mathbf{5}$ against Candida albicans and Aspergillus niger.
\end{abstract}

Keywords 2-Oxonicotinonitriles $\cdot$ Acyclic nucleosides $\cdot$ 2-Oxonicotinonitriles glycosides $\cdot$ Antibacterial activity Antifungal activity

\section{Introduction}

Emergence of antimicrobial resistance, especially multidrug bacterial resistance has posed worldwide problem in medicine $[1,2]$. The Center for Disease Control and Prevention (CDC) estimates that more than 2 million individuals become infected with drug-resistant bacteria annually, and greater than 23,000 individual dies as a result of those infections [3]. Gram-positive bacterium Staphylococcus aureus (Staph) is the causative agent of multitude of diseases including bacteremia or sepsis, pneumonia, endocarditis, and osteomyelitis. Staph became resistant to several antibiotics

Abdalla E. Hassan

habdallaa@aol.com

1 Applied Nucleic Acids Research Center, Zagazig University, Zagazig, Egypt

2 Chemistry Department, Faculty of Science, Zagazig University, Zagazig, Egypt such as methicillin-resistant Staphylococcus aureus (MRSA) [4-10], vancomycin-intermediate Staphylococcus aureus (VISA) [11, 12], and vancomycin-resistant Staphylococcus aureus (VRSA) [13-16]. Micrococci luteus are another Gram-positive bacterium that have been reported to cause pneumonia, meningitis associated with ventricular shunts, septic arthritis, bacteremia, peritonitis, endophthalmitis, and endocarditis. Micrococci have shown resistance to several antibiotics including streptomycin [17]. Pseudomonas aeruginosa is a Gram-negative bacterium that tends to infect people with immunodeficiency and those with indwelling catheters or on respirators. Infection with pseudomonas can lead to urinary tract infections (UTI), sepsis, pneumonia, and pharyngitis. Pseudomonas colonizes the lungs of patients with cystic fibrosis ( $\mathrm{CF}$ ) and contributes to the chronic progressive pulmonary disease and death rate in CF. $P$. aeruginosa is a Gram-negative bacterium that is intrinsically resistant to $\beta$-lactam-based antibiotics such as cephalothin and ampicillin [18-22]. Escherichia coli is the most common 
Gram-negative pathogen in human, and multiple antibiotic resistance $E$. coli strains have been reported [23-26].

2-Oxonicotinonitriles (2-ONN) ring is a core moiety in several biologically important compounds [27, 28]. This motif has been found in HIV-1-non-nucleoside reverse transcriptase inhibitors [27, 29, 30], non-nucleosides HBV inhibitors, and HCV NS5B polymerase inhibitors [31-35], anti-HSV-1 agents [36] (Fig. 1). In addition to their antiviral activity, 2-pyridinone derivatives are known to possess activity against wild type [37] and resistant bacterial strains [38]. Pyridone derivatives have also shown antifungal [39] and antiprotozoal activity [40, 41]. We have identified 2-ONN derivatives with significant anti-SARS and antiBacillus subtitis activity (1, 2; Fig. 1) [42]. The structural features of the 2-ONNs derivatives 1, 2 have two aryl substituents and a glycosyl/polar alkyl moieties at the 4 and 6 , and $\mathrm{N}-1$ positions, respectively. Though the molecular target for these 2-ONNs is still unknown, it is legitimate, and the structure activity relationship studies of these entities could provide useful antimicrobial agents. It is of interest to probe the effect of substituent at the C-6 and $N-1$ positions on the antimicrobial activities. Herein, we report on the synthesis of the 4-(p-methoxyphenyl)-6-cyclohexyl-2-ONNs derivatives 3-20 (Table 2; general structures I-II, Fig. 1) and their antimicrobial evaluations.

\section{Results and discussions}

\section{Chemistry}

Methods available in the literature for the synthesis of 4,6-disubstituted 2-ONN derivatives involve condensation
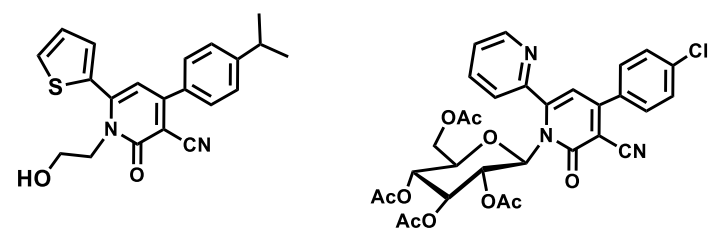

$$
\text { (1; anti-SARS-CoV, and }
$$
anti-Bacillis subtitis)

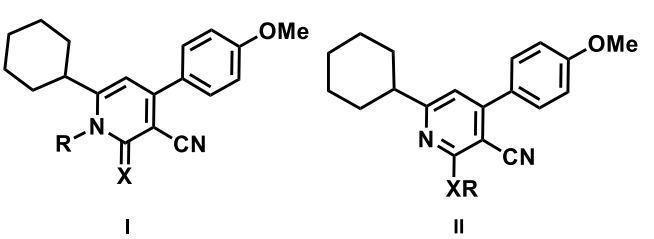

$X=O$ and $S$;

$R=$ Alkyl, Substituted alkyl, and Gylcosyl

Fig. 1 Structures of biologically active pyridone derivatives (1-2) and the newly synthesized 2-ONN (I, II) derivatives of 2-substituted acrylamide with enolates [43], one-pot four-component condensation reaction of aromatic aldehyde, ketone, ethyl cyanoacetate in the presence of ammonium acetate [44-48].The later procedure was adapted with minor modification for the synthesis of the 2-ONN derivative 3. Treatment of cyclohexyl methyl ketone with equimolar amounts of $p$-anisaldehyde, ethyl cyanoacetate, and $\mathrm{NH}_{4} \mathrm{OAc}$ in reflux EtOH gave the 2-ONN derivative 3 in good yield (Scheme 1).

A schematic representation of the reaction mechanism for the one-pot four-component reaction is illustrated in Fig. 2. The reaction may commence with aldol condensation providing the chalcone intermediate [I], which then reacts with the ethyl cyanoacetate to give the intermediate [II] which cyclizes with ammonia generated from ammonium acetate to give the corresponding dihydropyridine ring [III]. Oxidation of the intermediate [III] would give the pyridone derivative 3. 2-Pyridones are known to exist in two tautomeric forms: amide form and 2-hydroxypyridine form [49, 50], where the distribution ratio of these tautomers, among other factors, could affect the $\mathrm{N}-\mathrm{O} O$-alkylation selectivity at the 2 -ONN ring. Keto-enol tautomerism in 2-pyridone/2-hydroxypyridine is considered as an intramolecular hydrogen transfer reaction, where the hydrogen atom is transferred between the $N$ and the $O$ sites of the molecule through a transition state (A3) (Fig. 3). Spectroscopic data show the predominance of the keto form over the enol form. IR spectrum of 3 showed $\nu_{\mathrm{C}=\mathrm{O}} 1647 \mathrm{~cm}^{-1}$, and its ${ }^{13} \mathrm{C}$-NMR spectrum showed the signal for $(\mathrm{C} 2)$ at $161.8 \mathrm{ppm}$. Energies of the tautomers of the 2-ONN derivative 3 as well as that of the transition state were calculated via density functional theory (DFT) at $\omega B 97 X-D / 6-31 G^{*}$ and level of theory [51]. Energies listed in Table 1 coincide with the experimental results supporting the predominance of the keto form (A1) for 3 over the enol form (A3) tautomer (Fig. 4). The calculation results show that the energy of the transition state closer to the enol form compared with the keto form (Table 1). The local ionization potential and electrostatic potential maps are used to identify the nucleophilic or the electrophilic centers in a molecule.

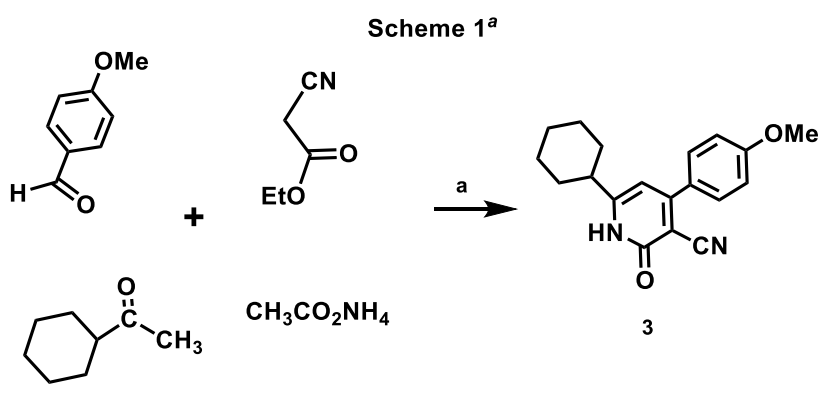

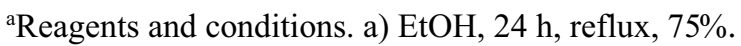

Scheme 1 Reagents and conditions. a EtOH, 24 h, reflux, 75\% 
Fig. 2 A plausible mechanism for the one-pot four-component synthesis of $\mathbf{3}$
Fig. 3 Optimized structures of 2-ONN tautomers and their transition state (TS) using DFT$\omega \mathrm{B} 97 \mathrm{X}-\mathrm{D}$ level of theory<smiles>CCOC(=O)CCCCCCCCCCC(N)=O</smiles><smiles>[R]c1cc([R2])c(C#N)c(O[Hg])n1</smiles>

Enol form<smiles></smiles>

TS

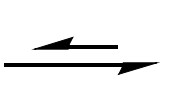<smiles>[R]c1cc([R2])n([14CH3])c(=O)c1C#N</smiles>

Table 1 Geometrical optimization of compound 3 s $\mathrm{s}$ tautomers and their TS

\begin{tabular}{llllll}
\hline Tautomer & Energy $(\mathrm{au})$ & $\begin{array}{l}\text { Relative energy } \\
(\mathrm{KJ} / \mathrm{mol})\end{array}$ & E HOMO $(\mathrm{eV})$ & E LUMO $(\mathrm{eV})$ & $\begin{array}{l}\text { Boltz- } \\
\text { mann } \\
\text { weight }\end{array}$ \\
\hline A1 & -995.714616 & 0.00 & -7.93 & -1.83 & 0.989 \\
A2 & -995.710322 & & -8.03 & -0.08 & 0.011 \\
A3 & -995.710341 & 11.22 & & & \\
\hline
\end{tabular}

The centers for electrophilic attack in $\mathbf{3}$ are $N^{1}$ and $O^{2}$ positions (Fig. 4).

The intrinsic low solubility of the diaryl-2-ONN derivatives in aqueous and most of the organic solvents creates a barrier for those entities to show biological activity. Branching of the diaryl-2-ONN derivatives with either transient or permanent solubilizing groups could overcome their low solubility problem. Glycosylation of small molecules, especially biologically active compounds, with specific mono- and disaccharides has been utilized to modulate their water solubility or their cellular uptake, and hence their pharmacological characteristics [52, 53]. In view of that, the 2-ONN derivative 3 was glycosylated with three glycosyl bromides under basic conditions (Table 2). Treatment of $\mathbf{3}$ with peracetylated $\alpha$-D-glucopyranosyl bromide [54] in the presence of $\mathrm{K}_{2} \mathrm{CO}_{3}$ in DMF at room temperature gave corresponding $\mathrm{N}$-glucoside derivative 4 in 57\% yield along with traces of the corresponding $O$-glycosyl derivative. In a similar manner, the $N$-lactoside derivative 6 was prepared by coupling of 3 with peracetylated 1- $\alpha$-D-lactosyl bromide [55] in $51 \%$ yield (Table 2). The $N^{1}$-glycosidation in $\mathbf{4}$ and $\mathbf{6}$ was confirmed by the presence of the amide $v_{\mathrm{C}=\mathrm{O}}$ signals in the range of $1644-1641 \mathrm{~cm}^{-1}$ in their IR spectra. The diaxial coupling between the anomeric proton and the $\mathrm{H}-2^{\prime}$ $\left(J_{1^{\prime}, 2^{\prime}}=7.86-8.82 \mathrm{~Hz}\right)$ confirms the $\beta$-configuration at the anomeric position of the 2-ONN glycosides (4-6). The observed preference of $\mathrm{N}$-glycosylation over $O$-glycosylation coincides with the DFT conclusion of the predominance of the keto form of the 2-ONN derivative 3, despite the steric hindrance at the C- 6 position. Treatment of the $\mathrm{N}$-glycosides 4 and $\mathbf{6}$ with triethylamine (TEA) in $\mathrm{H}_{2} \mathrm{O}$ / $\mathrm{MeOH}$ gave the corresponding free $\mathrm{N}$-glycosides 5 and $\mathbf{7}$, respectively, in good yields. Branching at the amide moiety 2-ONN derivatives with acyclic sugar mimics, alkyl or substituted alkyl derivatives, has resulted in better biological activity compared with the native 2-ONN [42]. Basecatalyzed coupling of $\mathbf{3}$ with 4-bromobutyl acetate gave a separable mixture of $\mathrm{N}$-butyl acetate derivative $\mathbf{8}$ and the corresponding $O$-butyl acetate derivative $\mathbf{1 0}$ in good yield (Table 2). Treatment of $\mathbf{3}$ with 2-bromomethoxyethyl 


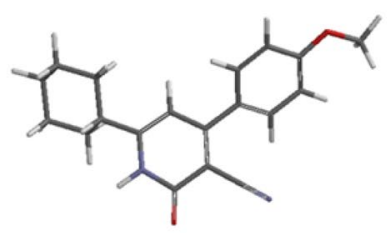

A1

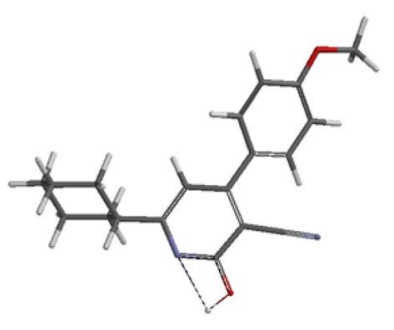

A2

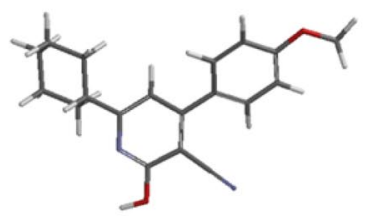

A3

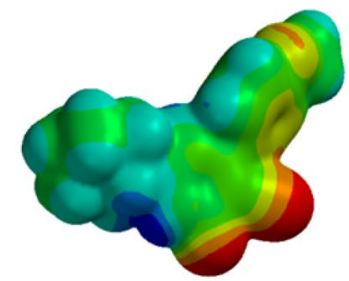

B1

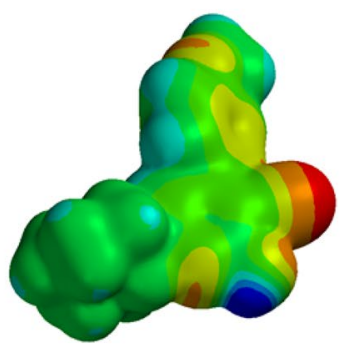

B2

B3

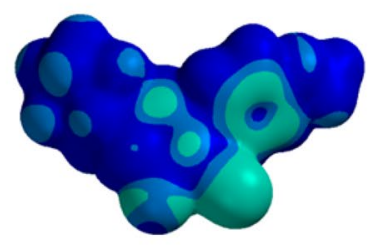

C1

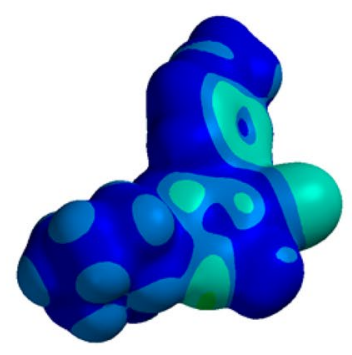

$\mathrm{C} 2$

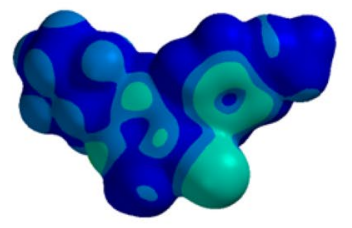

Fig. 4 A1, A3 Minimized energy for the tautomers of the 2-ONN derivative 3. B1, B2 Electrostatic potential map for the tautomers of the 2-ONN derivative 3. C1, C2 Local ionization map for the tautomers of the 2-ONN derivative 3

acetate gave a mixture of the corresponding $N$-acyclic nucleoside mimic $\mathbf{1 2}$ and the corresponding $O$-alkyl derivative $\mathbf{1 4}$ in good isolated yields (Table 2). The site of alkylation in the above mentioned reactions is determined by IR and ${ }^{13} \mathrm{C}$-NMR spectroscopy. For instance, the $N$-alkylation in 12 is supported by the presence of $\nu_{\mathrm{C}=\mathrm{O}}$ signal $\left(1647 \mathrm{~cm}^{-1}\right)$ in its IR spectrum and $170.3 \mathrm{ppm}(\mathrm{C} 2)$ signal in its ${ }^{13} \mathrm{C}$-NMR spectrum. Deacetylation of $\mathbf{8 , 1 0}$, 12, and 14 was performed with $\mathrm{Et}_{3} \mathrm{~N}$ in $\mathrm{MeOH} / \mathrm{H}_{2} \mathrm{O}$ to give $\mathbf{9}, \mathbf{1 1}, \mathbf{1 3}$, and 15, respectively, in good yields. Treatment of $\mathbf{3}$ with propargyl bromide under $\left(\mathrm{K}_{2} \mathrm{CO}_{3} /\right.$ acetone/reflux $)$ conditions gave a separable mixture of the $N$ - and $O$-propargyl derivatives $\mathbf{1 6}$ and $\mathbf{1 7}$ in good yields (Table 2). In a similar manner, treatment of $\mathbf{3}$ with allyl bromide under the same conditions gave a separable mixture of $N$-allyl and $O$-allyl derivatives $\mathbf{1 8}$ and 19, respectively, in good yields (Table 2). On the other hand, treatment of $\mathbf{3}$ with epichlorohydrin under the same conditions $\left(\mathrm{K}_{2} \mathrm{CO}_{3}\right.$ /acetone/reflux) none of the $O$-alkyl derivative was isolated, but the $N$-alkyl derivative 20 was isolated in $42 \%$ yield (Table 2).

\section{Biology}

\section{Evaluation of antimicrobial activities}

The new synthesized 2-ONN derivatives were screened in vitro for antibacterial activity against the Gram-positive bacteria: Staphylococcus aureus ATCC 6538 and M. luteus ATCC 10240 and Gram-negative bacteria: P. aeruginosa ATCC 9027 and Escherichia coli ATCC 10536. The antifungal activity of the 2-ONN derivatives was assessed against Candida albicans ATCC 10231 and Aspergillus niger ATCC 16404. Agar-diffusion method [42] was used to assess the antibacterial and antifungal activity of the tested compounds. The diameter of inhibition zone (IZ) of the bacterial and fungal growth in mm was used to assess the preliminary antimicrobial activity of the tested compounds [42]. 
Table 2 Glycosylation and alkylation of the 2-ONN derivative 3

$\begin{array}{llll}\text { Entry } & \text { Coupling agent } & \text { Product }^{\mathrm{b}, \mathrm{c}} & \text { Yield \% }\end{array}$

1

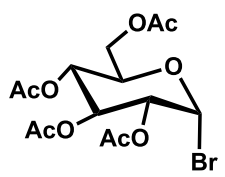

2
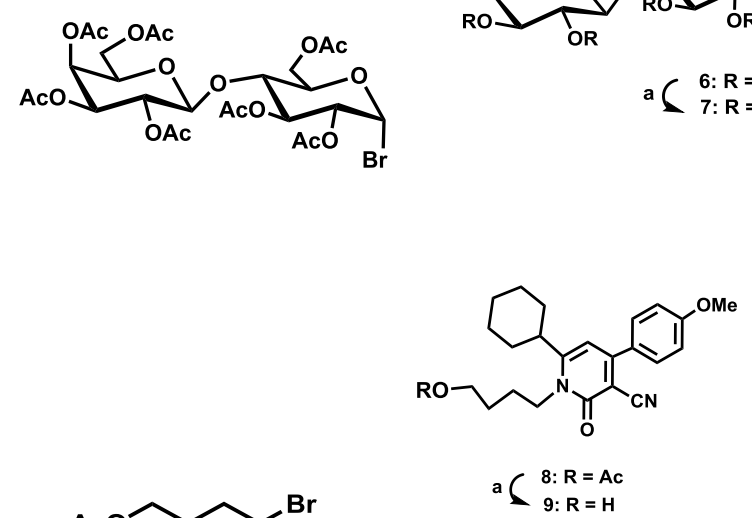

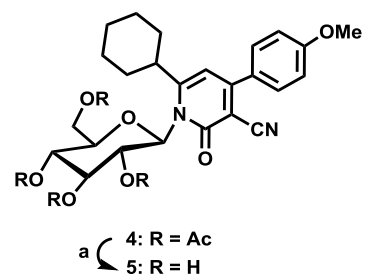

4: $57^{\mathrm{b}}$

5: 90

6: $49^{\mathrm{b}}$

7: 87

3
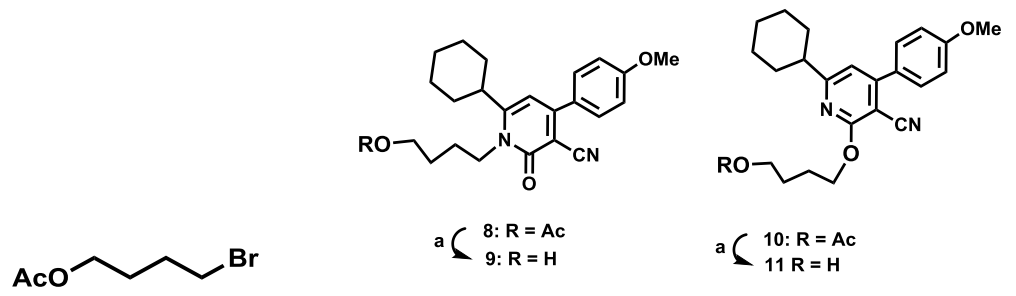

\section{8: $34^{\mathrm{c}}$ \\ 9: 80 \\ $1048^{\mathrm{c}}$}

11: 83

\section{Concentration of the tested samples was $4 \mathrm{mg} / \mathrm{mL}$, ND—not determined}

The parent 2-ONN derivative $\mathbf{3}$ showed significant activity against the Gram-negative bacteria: E. coli ATCC-10536 and $P$. aeruginosa ATCC-9027, while a moderate activity against the Gram-positive bacteria: S. aureus ATCC-6538 and M. luteus ATCC-10242, was observed (Table 3). Additionally, a significant antifungal activity was observed with the 2-ONN derivative $\mathbf{3}$, and its $\mathrm{N}$-glucoside derivatives 4 and 5 against Candida albicans and Aspergillus niger (Table 3). It is notable that weak to moderate antibacterial activity is observed with $O$-alkyl derivatives 11, 17, and 19 and the $N$-oxiranylmethyl derivative 20. The antifungal activity of selected number of the newly synthesized compounds was evaluated against Candida albicans ATCC 10231 and Aspergillus niger ATCC 16404. The parent compound 3, $N$-glucosyl acetate derivative 4 , its free derivative $\mathbf{5}$, and $N$-(2-hydroxyethoxy)methyl derivative $\mathbf{1 3}$ showed superior antifungal activity against both strains compared with nystatin. The data observed with compounds $4, \mathbf{5}, \mathbf{1 1}$,
13, 16-20 imply that branching of the parent 2-ONN derivative 3 with solubilizing groups affects the antimicrobial activity.

\section{Conclusions}

A one-pot four-component reaction was utilized to synthesize the 6-cyclohexyl-4-methoxy-2-ONN derivative $\mathbf{3}$ in good yield. DFT calculations of tautomeric forms of 3 show the predominance of the 2-keto form, (A1) over the enol form (A2). Branching of the amide moiety of $\mathbf{3}$ by glycosylation gave the corresponding $\mathrm{N}$-glycosides in good yields. Alkylation of $\mathbf{3}$ with several alkyl halides gave separable mixtures of the corresponding $N$ - and $O$-alkyl derivatives. Significant antibacterial activity was observed with 13, 16, and 18 against $S$. aureus ATCC-6538 and M. luteus ATCC10242, while the parent 2-ONN derivative 3 showed good activity against $E$. coli. On the other hand, a significant antifungal activity was observed in 2 -ONN derivative 3 , its $\mathrm{N}$-glucoside derivatives $\mathbf{4}$ and $\mathbf{5}$ against Candida albicans 
Table 2 (continued)

4

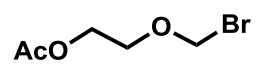

5<smiles>C#CCCl</smiles><smiles></smiles>

a $\begin{aligned} & \text { 12: } R=A c \\ & 13: R=H\end{aligned}$<smiles>COc1ccc(-c2cc(C3CCCCC3)nc(OCOCCO)c2C#N)cc1</smiles>

a $\begin{aligned} & \text { 14: } R=A c \\ & 15: R=H\end{aligned}$

12: $27^{\mathrm{c}}$

13: 83

14: $42^{\mathrm{c}}$

15: 87

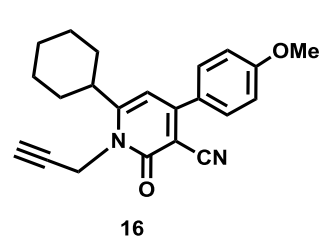<smiles>C#CCOc1nc(C2CCCCC2)cc(-c2ccc(OC)cc2)c1C#N</smiles>

16: $30^{\mathrm{c}}$

17: $29^{\mathrm{c}}$

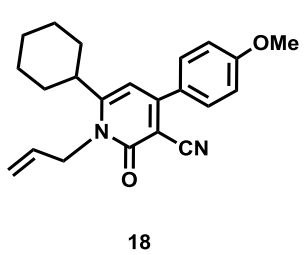

18

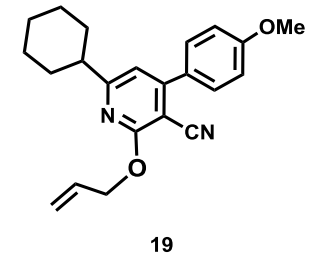

18: $32^{c}$

19: $39^{\mathrm{c}}$

6

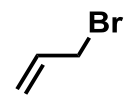

$V_{\mathrm{Cl}}$<smiles>N#Cc1c(-c2ccc(Cl)cc2)cc(C2CCCCC2)n(CC2CO2)c1=O</smiles>

20

${ }^{a} \mathrm{Et} 3 \mathrm{~N}, \mathrm{MeOH}, \mathrm{H} 2 \mathrm{O}$, r.t.

${ }^{\mathrm{b}} \mathrm{K} 2 \mathrm{CO} 3$, DMF, r.t.

${ }^{\mathrm{c}} \mathrm{K} 2 \mathrm{CO} 3$, acetone, reflux 
Table 3 Antibacterial and antifungal activity of selected 2-ONNs derivatives

\begin{tabular}{|c|c|c|c|c|c|c|}
\hline \multirow[t]{2}{*}{ Compd. } & \multicolumn{6}{|c|}{ Diameter of inhibition zone (mm) } \\
\hline & $\begin{array}{l}\text { S. aureus } \\
\text { ATCC } 6538\end{array}$ & $\begin{array}{l}\text { M. luteus } \\
\text { ATCC } \\
10240\end{array}$ & $\begin{array}{l}\text { P. aeruginosa } \\
\text { ATCC } 9027\end{array}$ & $\begin{array}{l}\text { E. coli } \\
\text { ATCC } \\
10536\end{array}$ & $\begin{array}{l}\text { Candida } \\
\text { albicans ATCC } \\
10231\end{array}$ & $\begin{array}{l}\text { Aspergillus } \\
\text { niger ATCC } \\
16404\end{array}$ \\
\hline 3 & 25 & 23 & 28 & 30 & 30 & 32 \\
\hline 4 & 30 & 34 & 22 & 23 & 30 & 32 \\
\hline 5 & 20 & 24 & 20 & 20 & 31 & 33 \\
\hline 6 & 29 & 30 & 20 & 19 & 20 & 21 \\
\hline 7 & 21 & 20 & 20 & 20 & 21 & 20 \\
\hline 8 & 26 & 29 & 20 & 20 & 25 & 28 \\
\hline 9 & 30 & 31 & 22 & 23 & 29 & 31 \\
\hline 10 & ND & ND & ND & ND & 32 & 35 \\
\hline 11 & 21 & 24 & 20 & 20 & 18 & 20 \\
\hline 13 & 37 & 39 & 20 & 22 & 30 & 33 \\
\hline 15 & 31 & 29 & 20 & 20 & ND & ND \\
\hline 16 & 34 & 33 & 22 & 19 & 27 & 29 \\
\hline 17 & 21 & 22 & 20 & 21 & ND & ND \\
\hline 18 & 36 & 39 & 21 & 22 & 30 & 32 \\
\hline 19 & 22 & 19 & 20 & 18 & ND & ND \\
\hline 20 & 24 & 25 & 20 & 23 & ND & ND \\
\hline Cefotaxime & 31 & 28 & 32 & 34 & & \\
\hline Nystatin & & & & & 25 & 20 \\
\hline DMF & & & & & & \\
\hline
\end{tabular}

Concentration of the tested samples was $4 \mathrm{mg} / \mathrm{mL}, N D$-not determined and Aspergillus niger. The results of this study provide hits for further optimizations for the discovery of potent antimicrobial agents.

\section{Experimental results and protocols}

\section{Material, instruments, and general considerations}

Starting materials and reagents were purchased from Acros-Organics, Alpha Aesar, and Sigma-Aldrich. 4-Bromobutyl acetate [56] and 2-bromomethoxyethyl acetate $[57,58]$ were prepared according to the literature procedures. Reaction's progress was monitored by TLC analysis using aluminum-backed plates pre-coated with Merck silica gel 60 F254. Column chromatography was carried out on Agela Technologies flash silica 40-60 mesh. Melting points were recorded on Electrothermal IA 9100 apparatus and were uncorrected. IR spectra ( $\mathrm{KBr}$ disk) were recorded on either a Pye Unicam Sp 3-300 or a Shimadzu FTIR $8101 \mathrm{PC}$ infrared spectrometer. ${ }^{1} \mathrm{H}$ and ${ }^{13} \mathrm{C}-\mathrm{NMR}$ spectra were recorded on Bruker $400 \mathrm{MHz}$ spectrometer. Chemical shifts $(\delta)$ were reported in ppm and were referenced to
TMS as a standard $(0.00 \mathrm{ppm})$. Chemical shifts for ${ }^{13} \mathrm{C}$ NMR were referenced relative to DMSO (39.50 ppm). Elemental microanalysis was recorded on PerkinElmer 240 analyzer. Analyses indicated by the symbols of the elements were within $0.4 \%$ of the theoretical values.

\section{Chemistry}

6-Cyclohexyl-4-(4-methoxyphenyl)-2-oxo-1,2-dihydropyrid ine-3-carbonitrile (3)

A mixture of 4-methoxybenzaldehyde $(6.80 \mathrm{~g}, 0.05 \mathrm{~mol})$, cyclohexyl methyl ketone $(6.31 \mathrm{~g}, 0.05 \mathrm{~mol})$, and ammonium acetate $(30.8 \mathrm{~g}, 0.4 \mathrm{~mol})$ in ethanol $(150 \mathrm{~mL})$ was refluxed for $48 \mathrm{~h}$. The reaction mixture was cooled to room temperature, and precipitate was filtered off, washed with ethanol, and dried under vacuum. The precipitate was recrystallized from a mixture of acetic acid/ethanol (1:5 $\mathrm{v}: \mathrm{v})$ to give 3 (11.56 g, 75\%) as a pale yellow crystals: m.p. 242-244 ${ }^{\circ} \mathrm{C}$; IR (KBr): $3286 \mathrm{~cm}^{-1}(\mathrm{NH}), 2036 \mathrm{~cm}^{-1}(\mathrm{CN})$ and $1647 \mathrm{~cm}^{-1}\left(\mathrm{C}=\mathrm{O}\right.$, amide); ${ }^{1} \mathrm{H}-\mathrm{NMR}$ (DMSO- $\left.d_{6}\right) \delta$ 1.23-1.84 $\left(11 \mathrm{H}, \mathrm{m}\right.$, cyclohexyl), $3.83\left(3 \mathrm{H}, \mathrm{s}, \mathrm{OCH}_{3}\right), 6.26$ (1H, s, H-5, pyridone), $7.07(2 \mathrm{H}, \mathrm{d}, \mathrm{Ar}-\mathrm{H}, J=8.7 \mathrm{~Hz})$, 
$7.60(2 \mathrm{H}, \mathrm{d}, \mathrm{Ar}-\mathrm{H}, J=8.7 \mathrm{~Hz}), 12.36(1 \mathrm{H}, \mathrm{s}, \mathrm{NH}) ;{ }^{13} \mathrm{C}-$ NMR (DMSO- $\left.d_{6}\right) \delta 25.03,25.66,30.80,41.64,55.39,88.9$, 104.0, 114.2, 116.9, 128.2, 129.8, 159.6, 159.8, 161.0, 161.8; Anal. Calcd for $\mathrm{C}_{19} \mathrm{H}_{20} \mathrm{~N}_{2} \mathrm{O}_{2}$ (308.37): C, 74.00; H, 6.54; N, 9.08. Found: C, 74.09; H, 6.55; N, 9.09.

\section{1-(2', $3^{\prime}, 4^{\prime}, 6^{\prime}$-Tetra-0-acetyl- $\beta$-D-glucopyranosyl)-6-cyclo- hexyl-4-(4-methoxyphenyl)-2-oxo-1,2-dihydropyridine-3-c arbonitrile (4)}

A mixture of $3(1 \mathrm{~g}, 3.24 \mathrm{mmol})$ and anhydrous $\mathrm{K}_{2} \mathrm{CO}_{3}$ $(0.45 \mathrm{~g}, 3.27 \mathrm{mmol})$ in dry DMF $(10 \mathrm{~mL})$ was stirred for $30 \mathrm{~min}$. at $0{ }^{\circ} \mathrm{C}$. 1 -Bromo-2,3,4,6-tetra- $O$-acetyl-D-glucopyranose [54] $(1.23 \mathrm{~g}, 3.0 \mathrm{mmol})$ was added to reaction mixture in portions, and the mixture was stirred overnight at room temperature. Aqueous $1 \mathrm{~N} \mathrm{NH}_{4} \mathrm{Cl}(5 \mathrm{~mL})$ was added, and the solvents were evaporated under reduced pressure. The residue was partitioned between EtOAc and $\mathrm{H}_{2} \mathrm{O}$; the organic phase was dried $\left(\mathrm{Na}_{2} \mathrm{SO}_{4}\right)$ and evaporated under reduced pressure. The residue was purified by a silica gel column chromatography (eluate: $10 \%$ EtOAc/hexanes) to give 4 (1.18 g,57\%yield) as a white powder: m.p. $184-186{ }^{\circ} \mathrm{C}$; IR (KBr) $2228 \mathrm{~cm}^{-1}(\mathrm{CN})$ and broad band at $1754 \mathrm{~cm}^{-1}(\mathrm{C}=\mathrm{O}$, acetoxy and amide $)$; ${ }^{1} \mathrm{H}-\mathrm{NMR}$ (DMSO- $d_{6}$ ) $\delta 1.30-1.85(10 \mathrm{H}, \mathrm{m}$, cyclohexyl), $1.97,1.98,2.00,2.02\left(12 \mathrm{H}, 4 \mathrm{~s}, 4 \mathrm{CH}_{3} \mathrm{CO}\right), 2.74(1 \mathrm{H}, \mathrm{t}$, H1-cyclohexyl, $J=2.80 \mathrm{~Hz}), 3.83\left(3 \mathrm{H}, \mathrm{s}, \mathrm{OC}_{3}\right), 4.01$ $\left(1 \mathrm{H}, \mathrm{d}, \mathrm{H}-6^{\prime}, J_{6^{\prime}, 5^{\prime}}=4.96 \mathrm{~Hz}, J_{6^{\prime}, 6^{\prime \prime}}=11.71 \mathrm{~Hz}\right), 4.18(1 \mathrm{H}$, $\left.\mathrm{dd}, \mathrm{H}-6^{\prime \prime}, J_{6^{\prime \prime}, 5^{\prime}}=5.60 \mathrm{~Hz}, J_{6^{\prime \prime}, 6^{\prime}}=5.60 \mathrm{~Hz}\right), 4.32(1 \mathrm{H}, \mathrm{m}$, $\left.\mathrm{H}-5^{\prime}\right), 5.03\left(1 \mathrm{H}, \mathrm{t}, \mathrm{H}-3^{\prime}, J_{3^{\prime}, 2^{\prime}}=9.60 \mathrm{~Hz}, J_{3^{\prime}, 4^{\prime}}=10.0 \mathrm{~Hz}\right)$, $5.17\left(1 \mathrm{H}, \mathrm{t}, \mathrm{H}-2^{\prime}, J_{2^{\prime}, 3^{\prime}}=4.80 \mathrm{~Hz}, J_{2^{\prime}, 1^{\prime}}=8.39 \mathrm{~Hz}\right), 5.60(1 \mathrm{H}$, t, H-4', $\left.J_{4^{\prime}, 5^{\prime}}=9.6 \mathrm{~Hz}, J_{4^{\prime}, 3^{\prime}}=9.6 \mathrm{~Hz}\right), 6.48\left(1 \mathrm{H}, \mathrm{d}, \mathrm{H}-1^{\prime}\right.$, $\left.J_{1^{\prime}, 2^{\prime}}=8.39 \mathrm{~Hz}\right), 7.12(1 \mathrm{H}, \mathrm{d}, \mathrm{Ar}-\mathrm{H}, J=8.8 \mathrm{~Hz}),, 7.21(1 \mathrm{H}$, s, pyridone-H-5), $7.64(1 \mathrm{H}, \mathrm{d}, \mathrm{Ar}-\mathrm{H}, J=8.8 \mathrm{~Hz}) ;{ }^{13} \mathrm{C}-\mathrm{NMR}$ $\left(\right.$ DMSO- $\left.d_{6}\right) \delta 20.18,20.24,20.27,20.32,25.36,25.67$, $25.72,31.25,55.34,61.85,68.13,70.06,71.45,72.02$, $91.22,93.24,114.2,114.3,116.7,127.5,130.0,155.5$, 160.7, 161.7, 168.3, 168.7, 169.2, 169.5, 169.8; Anal. Calcd for $\mathrm{C}_{33} \mathrm{H}_{38} \mathrm{~N}_{2} \mathrm{O}_{11}$ (638.66): C, 62.06; $\mathrm{H}, 6.00 ; \mathrm{N}, 4.39$. Found: C, 62.07; H, 6.01; N, 4.37.

\section{6-Cyclohexyl-1-( $\beta$-D-glucopyranosyl)-4-(4-methoxyphenyl)- 2-oxo-1,2-dihydropyridine-3-carbonitrile (5)}

A mixture of $4(0.63 \mathrm{~g}, 1.0 \mathrm{mmol}), \mathrm{Et}_{3} \mathrm{~N}(1 \mathrm{~mL})$, and $\mathrm{H}_{2} \mathrm{O}$ $(0.1 \mathrm{~mL})$ in $\mathrm{MeOH}(5 \mathrm{~mL})$ was stirred overnight at room temperature. The volatiles were evaporated under reduced pressure. The residue was repeatedly co-evaporated with $\mathrm{MeOH}$ until complete removal of $\mathrm{Et}_{3} \mathrm{~N}$, and the residue was crystallized from $95 \%$ ethanol gave $5(0.42 \mathrm{~g}, 90 \%$ yield $)$ as a white powder: m.p. $246-248{ }^{\circ} \mathrm{C}$; IR $(\mathrm{KBr}) 3427 \mathrm{~cm}^{-1}$ (4OH), $2217 \mathrm{~cm}^{-1}(\mathrm{CN})$ and $1644 \mathrm{~cm}^{-1}(\mathrm{C}=\mathrm{O}$, amide $) ;{ }^{1} \mathrm{H}-$ NMR (DMSO- $\left.d_{\sigma}-\mathrm{D}_{2} \mathrm{O}\right) \delta 1.16-1.84(10 \mathrm{H}, \mathrm{m}$, cyclohexyl),
$2.60(1 \mathrm{H}, \mathrm{t}$, cyclohexyl-H-1, $J=2.82 \mathrm{~Hz}), 3.1-3.5(6 \mathrm{H}, \mathrm{m}$, H-6', H-6"', H-5', H-4', H-3' and H-2'), 3.83 (3H, s, OC${ }_{3}$ ), $6.27\left(1 \mathrm{H}, \mathrm{d}, \mathrm{H}-1^{\prime}, J_{1^{\prime}, 2^{\prime}}=8.21 \mathrm{~Hz}\right), 6.98(1 \mathrm{H}, \mathrm{s}$, pyridoneH-5), $7.08(2 \mathrm{H}, \mathrm{d}, \mathrm{Ar}-\mathrm{H}, J=8.84 \mathrm{~Hz}), 7.61(2 \mathrm{H}, \mathrm{d}, \mathrm{Ar}-\mathrm{H}$, $J=8.84 \mathrm{~Hz}$ ); Anal. Calcd for $\mathrm{C}_{25} \mathrm{H}_{30} \mathrm{~N}_{2} \mathrm{O}_{7}(470.51)$ : C, 63.82; H, 6.43; N, 5.95. Found: C, 63.80; H, 6.42; N, 5.96.

\section{1-(2', $3^{\prime}, 4^{\prime}, 6^{\prime}$-Tetra-0-acetyl- $\beta$-D-galactopyrano} syl-(1 $\rightarrow 4)-\left(2^{\prime}, 3^{\prime}, 6^{\prime}\right.$-tri-0-acetyl- $\beta$-D-glucopyranosyl)-6cyclohexyl-4-(4-methoxyphenyl)-2-oxo-1,2-dihydropyridine-3-carbonitrile (6)

A mixture of $3(1 \mathrm{~g}, 3.24 \mathrm{mmol})$ and anhydrous $\mathrm{K}_{2} \mathrm{CO}_{3}$ $(0.45 \mathrm{~g}, 3.27 \mathrm{mmol})$ in dry DMF $(10 \mathrm{~mL})$ was stirred for $30 \mathrm{~min}$. at $0{ }^{\circ} \mathrm{C}$. 1 -Bromo- $2^{\prime}, 3^{\prime}, 4^{\prime}, 6^{\prime}$-tetra- $O$-acetyl- $\beta$ D-galactopyranosyl- $(1 \rightarrow 4)-\left(2^{\prime}, 3^{\prime}, 6^{\prime}\right.$-tri- $O$-acetyl- $\beta$-Dglucopyranose [55] $(1.85 \mathrm{~g}, 3.0 \mathrm{mmol})$ was added to the reaction mixture in portions, and the mixture was stirred for $14 \mathrm{~h}$ at room temperature. Aqueous $1 \mathrm{~N} \mathrm{NH}_{4} \mathrm{Cl}(5 \mathrm{~mL})$ was added, and the solvents were evaporated under reduced pressure. The residue was partitioned between EtOAc and $\mathrm{H}_{2} \mathrm{O}$; the organic phase was dried $\left(\mathrm{Na}_{2} \mathrm{SO}_{4}\right)$ and evaporated under reduced pressure. The residue was partitioned between EtOAc and $\mathrm{H}_{2} \mathrm{O}$; the organic phase was separated, dried $\left(\mathrm{Na}_{2} \mathrm{SO}_{4}\right)$, and evaporated under reduced pressure. The residue was purified by a silica gel column chromatography to give $6(1.47 \mathrm{~g}$, yield $49 \%)$ as a pale yellow powder: m.p. $150-152^{\circ} \mathrm{C}$. IR (KBr) $2227 \mathrm{~cm}^{-1}(\mathrm{CN})$ and broad band at $1752 \mathrm{~cm}^{-1}(\mathrm{C}=\mathrm{O}$, acetoxy $), 1644 \mathrm{~cm}^{-1}(\mathrm{C}=\mathrm{O}$, amide $)$ amidic groups); ${ }^{1} \mathrm{H}-\mathrm{NMR}$ (DMSO- $\left.d_{6}\right) \delta 1.12-1.85(10 \mathrm{H}, \mathrm{m}$, cyclohexyl), 1.94, 1.96, 1.97, 2.01, 2.03, 2.05, $2.06(21 \mathrm{H}$, $\left.7 \mathrm{~s}, 7 \mathrm{CH}_{3} \mathrm{CO}\right), 2.73(1 \mathrm{H}, \mathrm{t}$, cyclohexyl-H-1,J=2.86 Hz), 3.83 ( $\left.3 \mathrm{H}, \mathrm{s}, \mathrm{OCH}_{3}\right), 4.03-4.08$ (3H, m, H-2'b, H-6'a and H-6'b), $4.15\left(1 \mathrm{H}, \mathrm{dd}, \mathrm{H}-6^{\prime \prime} \mathrm{a}, J_{6^{\prime \prime} \mathrm{a}, 6^{\prime} \mathrm{a}}=11.49, J_{6^{\prime \prime} \mathrm{a}, 5^{\prime} \mathrm{a}}=5.61 \mathrm{~Hz}\right), 4.26$ $\left(1 \mathrm{H}, \mathrm{m}, \mathrm{H}-5^{\prime} \mathrm{b}\right), 5.03\left(1 \mathrm{H}, \mathrm{dd}, \mathrm{H}-6^{\prime \prime} \mathrm{b}, J_{6^{\prime \prime} \mathrm{b}, 5^{\prime} \mathrm{b}}=6.32 \mathrm{~Hz}\right)$, $5.07\left(1 \mathrm{H}, \mathrm{m}, \mathrm{H}-5^{\prime} \mathrm{a}\right), 5.19\left(1 \mathrm{H}, \mathrm{dd}, \mathrm{H}-1^{\prime} \mathrm{b}, J_{1^{\prime} \mathrm{b}, 2^{\prime} \mathrm{b}}=7.86 \mathrm{~Hz}\right)$, $5.22\left(1 \mathrm{H}, \mathrm{dd}, \mathrm{H}-4^{\prime} \mathrm{b}, J_{4^{\prime} \mathrm{b}, 3^{\prime} \mathrm{b}}=3.42, J_{4^{\prime} \mathrm{b}, 5^{\prime} \mathrm{b}}=3.82 \mathrm{~Hz}\right), 5.36$ $\left(1 \mathrm{H}, \mathrm{dd}, \mathrm{H}-2^{\prime} \mathrm{a}, J_{2^{\prime} \mathrm{a}, 1^{\prime} \mathrm{a}}=8.97, J_{2^{\prime} \mathrm{a}, 3^{\prime} \mathrm{a}}=8.40 \mathrm{~Hz}\right), 5.38(1 \mathrm{H}$, $\left.\mathrm{dd}, \mathrm{H}-4^{\prime} \mathrm{a}, J_{4^{\prime} \mathrm{a}, 3^{\prime} \mathrm{a}}=9.15, J_{4^{\prime} \mathrm{a}, 5^{\prime} \mathrm{a}}=6.90 \mathrm{~Hz}\right), 5.64(1 \mathrm{H}, \mathrm{d}$, $\left.\mathrm{H}-3^{\prime} \mathrm{b}, J_{3^{\prime} \mathrm{b}, 4^{\prime} \mathrm{b}}=3.45 \mathrm{~Hz}\right), 6.44\left(1 \mathrm{H}, \mathrm{d}, \mathrm{H}-3^{\prime} \mathrm{a}, J_{3^{\prime} \mathrm{a}, 2^{\prime} \mathrm{a}}=6.40 \mathrm{~Hz}\right)$, $6.56\left(1 \mathrm{H}, \mathrm{d}, \mathrm{H}-1^{\prime} \mathrm{a}, J_{1^{\prime} \mathrm{a}, 2^{\prime} \mathrm{a}}=8.82 \mathrm{~Hz}\right), 7.12(1 \mathrm{H}, \mathrm{d}, \mathrm{Ar}-\mathrm{H}$, $J=7.99 \mathrm{~Hz}), 7.23(1 \mathrm{H}$, s, pyridone-H-5), $7.63(1 \mathrm{H}, \mathrm{d}, \mathrm{Ar}-\mathrm{H}$, $J=8.39 \mathrm{~Hz}$ ); Anal. Calcd for $\mathrm{C}_{45} \mathrm{H}_{54} \mathrm{~N}_{2} \mathrm{O}_{19}(926.91)$ : C, 58.31; H, 5.87; N, 3.02. Found: C, 58.30; H, 5.88; N, 3.02.

1 - $(\beta$-D-galactopyranosyl- $(1 \rightarrow 4)-(\beta-D$-glucopyranosyl)-6-cyclohexyl-4-(4-methoxyphenyl)-2-oxo-1,2dihydropyridine-3-carbonitrile (7). A mixture of $6(0.92 \mathrm{~g}$, $1.0 \mathrm{mmol}), \mathrm{Et}_{3} \mathrm{~N}(1 \mathrm{~mL})$, and $\mathrm{H}_{2} \mathrm{O}(0.1 \mathrm{~mL})$ in $\mathrm{MeOH}$ $(5 \mathrm{~mL})$ was stirred overnight at room temperature. The volatiles were evaporated under reduced pressure. The residue was repeatedly co-evaporated with $\mathrm{MeOH}$ until complete removal of $\mathrm{Et}_{3} \mathrm{~N}$, and the residue was crystallized from $95 \%$ ethanol to give 7 ( $0.55 \mathrm{~g}, 87 \%$ yield) as a pale yellow 
powder: m.p. $220-222{ }^{\circ} \mathrm{C}$; IR (KBr) $3412 \mathrm{~cm}^{-1}(7 \mathrm{OH})$, $2224 \mathrm{~cm}^{-1}(\mathrm{CN})$ and $1641 \mathrm{~cm}^{-1}\left(\mathrm{C}=\mathrm{O}\right.$, amide); ${ }^{1} \mathrm{H}-\mathrm{NMR}$ (DMSO- $\left.d_{6} / \mathrm{D}_{2} \mathrm{O}\right) \delta 1.16-1.89(10 \mathrm{H}, \mathrm{m}$, cyclohexyl), 2.83 $(1 \mathrm{H}, \mathrm{t}, \mathrm{H}-1$ cyclohexyl, $J=2.83 \mathrm{~Hz}), 3.85\left(3 \mathrm{H}, \mathrm{s}, \mathrm{OCH}_{3}\right)$, 3.94-4.12 (3H, m, H-2'b, H-6'a and H-6'b), $4.16(1 \mathrm{H}$, dd, H-6"a, $\left.J_{6^{\prime \prime} \mathrm{a}, 6^{\prime} \mathrm{a}}=11.59, J_{6^{\prime \prime} \mathrm{a}, 5^{\prime} \mathrm{a}}=5.64 \mathrm{~Hz}\right), 4.22(1 \mathrm{H}, \mathrm{m}$, H-5'b), $4.56\left(1 \mathrm{H}, \mathrm{dd}, \mathrm{H}-6^{\prime \prime} \mathrm{b}, J_{6^{\prime \prime} \mathrm{b}, 5^{\prime} \mathrm{b}}=6.35 \mathrm{~Hz}\right), 4.98(1 \mathrm{H}$, m, H-5'a), $5.14\left(1 \mathrm{H}, \mathrm{dd}, \mathrm{H}-1^{\prime} \mathrm{b}, J_{1^{\prime} \mathrm{b}, 2^{\prime} \mathrm{b}}=7.76 \mathrm{~Hz}\right), 5.20$ $\left(1 \mathrm{H}, \mathrm{dd}, \mathrm{H}-4^{\prime} \mathrm{b}, J_{4^{\prime} \mathrm{b}, 3^{\prime} \mathrm{b}}=3.62, J_{4^{\prime} \mathrm{b}, 5^{\prime} \mathrm{b}}=3.86 \mathrm{~Hz}\right), 5.31(1 \mathrm{H}$, $\left.\mathrm{dd}, \mathrm{H}-2^{\prime} \mathrm{a}, J_{2^{\prime} \mathrm{a}, 1^{\prime} \mathrm{a}}=8.87 \mathrm{~Hz}, J_{2^{\prime} \mathrm{a}, 3^{\prime} \mathrm{a}}=8.44 \mathrm{~Hz}\right), 5.34(1 \mathrm{H}, \mathrm{dd}$, $\left.\mathrm{H}-4^{\prime} \mathrm{a}, J_{4^{\prime} \mathrm{a}, 3^{\prime} \mathrm{a}}=9.05, J_{4^{\prime} \mathrm{a}, 5^{\prime} \mathrm{a}}=6.56 \mathrm{~Hz}\right), 5.62\left(1 \mathrm{H}, \mathrm{d}, \mathrm{H}-3^{\prime} \mathrm{b}\right.$, $\left.J_{3^{\prime} \mathrm{b}, 4^{\prime} \mathrm{b}}=3.55 \mathrm{~Hz}\right), 6.24\left(1 \mathrm{H}, \mathrm{d}, \mathrm{H}-3^{\prime} \mathrm{a}, J_{3^{\prime} \mathrm{a}, 2^{\prime} \mathrm{a}}=6.43 \mathrm{~Hz}\right)$, $6.76\left(1 \mathrm{H}, \mathrm{d}, \mathrm{H}-1^{\prime} \mathrm{a}, J_{1^{\prime} \mathrm{a}, 2^{\prime} \mathrm{a}}=8.80 \mathrm{~Hz}\right), 7.15(1 \mathrm{H}, \mathrm{d}, \mathrm{Ar}-\mathrm{H}$, $J=7.99 \mathrm{~Hz}), 7.25(1 \mathrm{H}, \mathrm{s}$, pyridone-H-5), $7.69(1 \mathrm{H}, \mathrm{d}, \mathrm{Ar}-\mathrm{H}$, $J=8.39 \mathrm{~Hz}$ ); Anal. Calcd for $\mathrm{C}_{31} \mathrm{H}_{40} \mathrm{~N}_{2} \mathrm{O}_{12}$ (632.66): C, 58.85; H, 6.37; N, 4.43. Found: C, 58.86; H, 6.37; N, 4.43.

4-(3-Cyano-6-cyclohexyl-4-(4-methoxyphenyl)-2oxopyridin-1 $(2 \mathrm{H})-y l)$ butyl acetate (8) and 4-((3-Cyano6-cyclohexyl-4-(4-methoxyphenyl)pyridin-2-yl)oxy)butyl acetate (10). A mixture of $\mathbf{3}(1 \mathrm{~g}, 3.24 \mathrm{mmol})$ with 4-bromobutyl acetate $(0.58 \mathrm{~g}, 3.0 \mathrm{mmol})$ was added to dry acetone $(10 \mathrm{~mL})$ in the presence of anhydrous $\mathrm{K}_{2} \mathrm{CO}_{3}(0.45 \mathrm{~g}$, $3.27 \mathrm{mmol}$ ); the reaction mixture was heated for $15 \mathrm{~h}$ at reflux temperature. The reaction mixture was cooled to room temperature, and the insoluble were removed by filtration. The filtrate was evaporated under reduced pressure and then the formed solid product was separated by silica gel chromatography (eluate; $\mathrm{CH}_{2} \mathrm{Cl}_{2}$ to $5 \%$ EtOAc/ $\mathrm{CH}_{2} \mathrm{Cl}_{2}$ ) to give $\mathbf{8}(0.44 \mathrm{~g}, 32 \%$ yield) as a pale yellow oil and $\mathbf{1 0}$ ( $0.65 \mathrm{~g}, 48 \%$ yield) as a pale yellow oil. Spectral and analytical data for 8: IR (KBr): $2219 \mathrm{~cm}^{-1}(\mathrm{CN}), 1734 \mathrm{~cm}^{-1}$ $(\mathrm{C}=\mathrm{O}$, acetoxy $)$ and $1644 \mathrm{~cm}^{-1}(\mathrm{C}=\mathrm{O}$, amide $) ;{ }^{1} \mathrm{H}$ NMR (DMSO-d $\left.{ }_{6}\right) \delta 1.13-1.86\left(14 \mathrm{H}, \mathrm{m}\right.$, cyclohexyl, $\mathrm{C}_{2 \mathrm{~b}}$, and $\left.\mathrm{CH}_{2 \mathrm{c}}\right), 2.01\left(3 \mathrm{H}, \mathrm{s}, \mathrm{COCH}_{3}\right), 2.55(1 \mathrm{H}, \mathrm{t}, \mathrm{H}-1$ cyclohexyl, $J=2.82 \mathrm{~Hz}), 3.83\left(3 \mathrm{H}, \mathrm{s}, \mathrm{OCH}_{3}\right), 4.07\left(\mathrm{t}, 1 \mathrm{H}, \underline{\mathrm{C}}_{2 \mathrm{a}}, J=5.60\right.$, $5.95 \mathrm{~Hz}), 4.46\left(\mathrm{t}, 1 \mathrm{H}, \mathrm{CH}_{2 \mathrm{~d}}, J=7.93,12.81 \mathrm{~Hz}\right), 6.41(\mathrm{~s}, 1 \mathrm{H}$, H-5 pyridone), $7.09(2 \mathrm{H}, \mathrm{d}, \mathrm{Ar}-\mathrm{H}, J=8.00 \mathrm{~Hz}), 7.63(2 \mathrm{H}, \mathrm{d}$, $\mathrm{Ar}-\mathrm{H}, J=9.19 \mathrm{~Hz}$ ); Anal. Calcd for $\mathrm{C}_{25} \mathrm{H}_{30} \mathrm{~N}_{2} \mathrm{O}_{4}(422.52)$ : C, 71.07; H, 7.16; N, 6.63. Found: C, 71.08; H, 7.19; N, 6.66. Spectral and analytical data for 10: IR $(\mathrm{KBr}) 2223 \mathrm{~cm}^{-1}$ (CN) and $1734 \mathrm{~cm}^{-1}\left(\mathrm{C}=\mathrm{O}\right.$, acetoxy); ${ }^{1} \mathrm{H}-\mathrm{NMR}\left(\mathrm{DMSO}-d_{6}\right)$ 81.12-1.87 (14H, m, cyclohexyl, $\underline{\mathrm{C}}_{2 \mathrm{~b}}$, and $\left.\mathrm{C}_{2 \mathrm{c}}\right), 1.97(3 \mathrm{H}$, $\left.\mathrm{s}, \mathrm{COC}_{3}\right), 2.62(1 \mathrm{H}, \mathrm{t}$, cyclohexyl-H-1, $J=2.81 \mathrm{~Hz}), 3.81$ $\left(3 \mathrm{H}, \mathrm{s}, \mathrm{OCH}_{3}\right), 4.05\left(1 \mathrm{H}, \mathrm{t}, \mathrm{CH}_{2 \mathrm{a}}, J=6.01 \mathrm{~Hz}\right), 4.45(1 \mathrm{H}, \mathrm{t}$, $\left.\mathrm{CH}_{2}(\mathrm{~d}), J=5.62 \mathrm{~Hz}\right), 7.03(1 \mathrm{H}, \mathrm{s}$, pyridone-H-5), $7.07(2 \mathrm{H}$, d, Ar-H, $J=8.69 \mathrm{~Hz}), 7.58(2 \mathrm{H}, \mathrm{d}, \mathrm{Ar}, J=8.69 \mathrm{~Hz})$;Anal. Calcd. for $\mathrm{C}_{25} \mathrm{H}_{30} \mathrm{~N}_{2} \mathrm{O}_{4}$ (422.52): C, 71.07; H, 7.16; N, 6.63. Found: C, 71.09; H, 7.15; N, 6.64.

6-Cyclohexyl-1-(4-hydroxybutyl)-4-(4-methoxyphenyl)-2-oxo-1,2-dihydropyridine-3-carbonitrile (9). A mixture of $8(0.5 \mathrm{~g}, 1.18 \mathrm{mmol}), \mathrm{Et}_{3} \mathrm{~N}(1 \mathrm{~mL})$, and $\mathrm{H}_{2} \mathrm{O}(0.1 \mathrm{~mL})$ in $\mathrm{MeOH}(5 \mathrm{~mL})$ was stirred overnight at room temperature. The volatiles were evaporated under reduced pressure. The residue was repeatedly co-evaporated with $\mathrm{MeOH}$ until complete removal of $\mathrm{Et}_{3} \mathrm{~N}$, and the residue was purified by silica gel column chromatography (eluate $10 \% \mathrm{MeOH} / \mathrm{CH}_{2} \mathrm{Cl}_{2}$ ) to give 9 (0.36 g, $80 \%$ yield) as a pale yellow syrup: IR $(\mathrm{KBr}) 3428 \mathrm{~cm}^{-1}(\mathrm{OH}), 2221 \mathrm{~cm}^{-1}(\mathrm{CN})$ and $1642 \mathrm{~cm}^{-1}$ $\left(\mathrm{C}=\mathrm{O}\right.$, amide); ${ }^{1} \mathrm{H}-\mathrm{NMR}$ (DMSO- $\left.d_{6} / \mathrm{D}_{2} \mathrm{O}\right) \delta 1.22-1.87$ (14H, m, cyclohexyl, and $\mathrm{C}_{2 \mathrm{~b}}$, and $\left.\mathrm{C}_{2 \mathrm{c}}\right), 2.68(1 \mathrm{H}, \mathrm{t}$, $\mathrm{H} 1$-cyclohexyl, $J=2.80 \mathrm{~Hz}), 3.44\left(2 \mathrm{H}, \mathrm{t}, \mathrm{CH}_{2 \mathrm{~d}}, J=6.42 \mathrm{~Hz}\right)$, $3.81\left(3 \mathrm{H}, \mathrm{s}, \mathrm{OC}_{3}\right), 4.43\left(2 \mathrm{H}, \mathrm{t}, \mathrm{CH}_{2 \mathrm{a}}, J=6.82 \mathrm{~Hz}\right), 7.02$ (1H, s, H-5 pyridone), $7.08(2 \mathrm{H}, \mathrm{d}, \mathrm{Ar}-\mathrm{H}, J=7.64 \mathrm{~Hz}), 7.59$ $(2 \mathrm{H}, \mathrm{d}, \mathrm{Ar}-\mathrm{H}, J=7.64 \mathrm{~Hz})$; Anal. Calcd for $\mathrm{C}_{23} \mathrm{H}_{28} \mathrm{~N}_{2} \mathrm{O}_{3}$ (380.48): C, 72.60; H, 7.42; N, 7.36. Found: C, 72.63; H, $7.45 ; \mathrm{N}, 7.35$.

6-Cyclohexyl-2-(4-hydroxybutoxy)-4-(4-methoxyphenyl) nicotinonitrile (11). A mixture of $\mathbf{1 0}(0.42 \mathrm{~g}, 1.0 \mathrm{mmol})$, $\mathrm{Et}_{3} \mathrm{~N}(1 \mathrm{~mL})$, and $\mathrm{H}_{2} \mathrm{O}(0.1 \mathrm{~mL})$ in $\mathrm{MeOH}(5 \mathrm{~mL})$ was stirred overnight at room temperature. The volatiles were evaporated under reduced pressure. The residue was repeatedly coevaporated with $\mathrm{MeOH}$ until complete removal of $\mathrm{Et}_{3} \mathrm{~N}$, and the residue was purified by a silica gel column chromatography (eluate: $\left.7 \% \mathrm{MeOH} / \mathrm{CH}_{2} \mathrm{Cl}_{2}\right)$ to give $\mathbf{1 1}(0.31 \mathrm{~g}, 83 \%$ yield) as a colorless syrup: IR (KBr) $3426 \mathrm{~cm}^{-1}(\mathrm{OH})$ and $2222 \mathrm{~cm}^{-1}(\mathrm{CN}) ;{ }^{1} \mathrm{H}-\mathrm{NMR}$ (DMSO- $\left.d_{6}\right) \delta 1.33-1.89(14 \mathrm{H}$, $\mathrm{m}$, cyclohexyl, $\mathrm{C}_{2 \mathrm{~b}}$, and $\left.\mathrm{C}_{2 \mathrm{c}}\right), 2.68(1 \mathrm{H}, \mathrm{t}, \mathrm{H}-1$ cyclohexyl, $J=2.80 \mathrm{~Hz}), 3.08\left(1 \mathrm{H}, \mathrm{d}\right.$, exchangeable with $\mathrm{D}_{2} \mathrm{O}, \mathrm{OH}$, $J=7.21 \mathrm{~Hz}), 3.43\left(2 \mathrm{H}, \mathrm{t}, \mathrm{CH}_{2 \mathrm{~d}}, J=5.62, J=6.40 \mathrm{~Hz}\right), 3.83$ $\left.\left(3 \mathrm{H}, \mathrm{s}, \mathrm{OC}_{3}\right), 4.43\left(2 \mathrm{H}, \mathrm{t}, \mathrm{C}_{2 \mathrm{a}}\right), J=6.42, J=6.01 \mathrm{~Hz}\right)$, 7.04 (1H, s, H-5pyridone), $7.08(2 \mathrm{H}, \mathrm{d}, \mathrm{Ar}-\mathrm{H}, J=8.46 \mathrm{~Hz}$ ), $7.60(2 \mathrm{H}, \mathrm{d}, \mathrm{Ar}-\mathrm{H}, J=8.46 \mathrm{~Hz})$; Anal. Calcd for $\mathrm{C}_{23} \mathrm{H}_{28} \mathrm{~N}_{2} \mathrm{O}_{3}$ (380.48): C, 72.60; H, 7.42; N, 7.36. Found: C, 72.65; H, $7.44 ; \mathrm{N}, 7.39$.

2-((3-Cyano-6-cyclohexyl-4-(4-methoxyphenyl)-2oxopyridin-1 $(2 \mathrm{H})-y l)$ methoxy)ethyl acetate (12) and 2-(((3-Cyano-6-cyclohexyl-4-(4-methoxyphenyl)pyridin2-yl)oxy)methoxy)ethyl acetate (14). A mixture of $\mathbf{3}(1 \mathrm{~g}$, $3.24 \mathrm{mmol})$ with (2-acetoxyethoxy)methyl bromide $(0.59 \mathrm{~g}$, $3.0 \mathrm{mmol})$ was added to dry acetone $(10 \mathrm{~mL})$ in the presence of anhydrous $\mathrm{K}_{2} \mathrm{CO}_{3}(0.45 \mathrm{~g}, 3.27 \mathrm{mmol})$; the reaction mixture was heated for $15 \mathrm{~h}$ under reflux temperature. The reaction mixture was cooled to room temperature, and the inorganic residue was removed by filtration. The solvent was evaporated under reduced pressure and then the residue was purified by silica gel chromatography (eluate: $\mathrm{CH}_{2} \mathrm{Cl}_{2}$ to $3 \%$ EtoAc in $\left.\mathrm{CH}_{2} \mathrm{Cl}_{2}\right)$ to give $\mathbf{1 2}(0.37 \mathrm{~g}, 27 \%$ yield) as a colorless oil and $\mathbf{1 4}(0.57 \mathrm{~g}, 42 \%$ yield $)$ as a pale yellow syrup. Spectral and analytical data for 12: IR (KBr) $2222 \mathrm{~cm}^{-1}(\mathrm{CN}), 1735 \mathrm{~cm}^{-1}(\mathrm{C}=\mathrm{O}$, acetoxy) and $1647 \mathrm{~cm}^{-1}\left(\mathrm{C}=\mathrm{O}\right.$, amide); ${ }^{1} \mathrm{H}-\mathrm{NMR}$ (DMSO- $\left.d_{6}\right) \delta 1.58-1.84$ $\left(10 \mathrm{H}, \mathrm{m}\right.$, cyclohexyl), $2.01\left(3 \mathrm{H}, \mathrm{s}, \mathrm{COCH}_{3}\right), 2.26(1 \mathrm{H}, \mathrm{t}$, H1-cyclohexyl-, $J=2.84 \mathrm{~Hz}), 3.79\left(3 \mathrm{H}, \mathrm{s}, \mathrm{OCH}_{3}\right), 4.06(3 \mathrm{H}$, t, $\left.\mathrm{C}_{2}(\mathrm{c}), J=4.81,6.00 \mathrm{~Hz}\right), 4.47\left(3 \mathrm{H}, \mathrm{t}, \mathrm{C}_{2}(\mathrm{~d}), J=5.63\right.$, $4.81 \mathrm{~Hz}), 4.60\left(2 \mathrm{H}, \mathrm{s}, \mathrm{CH}_{2}(\mathrm{a})\right), 7.05(1 \mathrm{H}, \mathrm{s}, \mathrm{H} 5$-pyridone), $7.63(2 \mathrm{H}, \mathrm{d}, \mathrm{Ar}-\mathrm{H}, J=8.82 \mathrm{~Hz}), 7.90(2 \mathrm{H}, \mathrm{d}, \mathrm{Ar}-\mathrm{H}$, 
$J=8.82 \mathrm{~Hz}$ ) $;{ }^{13} \mathrm{C}$-NMR (DMSO- $d_{6}$ ) $\delta 18.80,20.67,24.10$, $25.15,28.84,30.62,60.32,63.32,66.91,99.63,116.0,120.2$, 127.9, 128.1, 129.3, 130.8, 133.2, 134.5, 159.4, 170.3; Anal. Calcd for $\mathrm{C}_{24} \mathrm{H}_{28} \mathrm{~N}_{2} \mathrm{O}_{5}$ (424.49): C, 67.91; H, 6.65; N, 6.60. Found: C, 67.94; H, 6.63; N, 6.60. Spectral and analytical data for 14: IR (KBr) $2222 \mathrm{~cm}^{-1}(\mathrm{CN})$ and $1740 \mathrm{~cm}^{-1}$ $\left(\mathrm{C}=\mathrm{O}\right.$, acetoxy); ${ }^{1} \mathrm{H}-\mathrm{NMR}\left(\mathrm{DMSO}_{\mathrm{d}}\right) \mathrm{d}_{6} \delta 1.47-1.76(10 \mathrm{H}, \mathrm{m}$, cyclohexyl), $1.98\left(3 \mathrm{H}, \mathrm{s}, \mathrm{COC} \underline{\mathrm{H}}_{3}\right), 2.65(1 \mathrm{H}, \mathrm{t}$, cyclohexyl$\mathrm{H}-1, J=2.85 \mathrm{~Hz}), 3.83\left(3 \mathrm{H}, \mathrm{s}, \mathrm{OC}_{3}\right), 4.05\left(2 \mathrm{H}, \mathrm{t}, \mathrm{CH}_{2}(\mathrm{c})\right.$, $J=6.42 \mathrm{~Hz}), 4.44\left(2 \mathrm{H}, \mathrm{t}, \mathrm{CH}_{2 \mathrm{~d}}, J=5.91 \mathrm{~Hz}\right), 4.64(2 \mathrm{H}, \mathrm{s}$, $\mathrm{CH}_{2}$ (a)), $7.03(1 \mathrm{H}, \mathrm{s}$, pyridone-H-5), $7.08(2 \mathrm{H}, \mathrm{d}, \mathrm{Ar}-\mathrm{H}$, $J=8.83 \mathrm{~Hz}), 7.62(2 \mathrm{H}, \mathrm{d}, \mathrm{Ar}-\mathrm{H}, J=8.83 \mathrm{~Hz})$; Anal. Calcd for $\mathrm{C}_{24} \mathrm{H}_{28} \mathrm{~N}_{2} \mathrm{O}_{5}$ (424.49): C, 67.91; H, 6.65; N, 6.60. Found: $\mathrm{C}, 67.89 ; \mathrm{H}, 6.64 ; \mathrm{N}, 6.60$.

6-Cyclohexyl-1-((2-hydroxyethoxy)methyl)-4-(4methoxyphenyl)-2-oxo-1,2-dihydropyridine-3-carbonitrile (13). A mixture of $12(0.42 \mathrm{~g}, 1.0 \mathrm{mmol}), \mathrm{Et}_{3} \mathrm{~N}(1 \mathrm{~mL})$, and $\mathrm{H}_{2} \mathrm{O}(0.1 \mathrm{~mL})$ in $\mathrm{MeOH}(5 \mathrm{~mL})$ was stirred overnight at room temperature. The volatiles were evaporated under reduced pressure. The residue was repeatedly co-evaporated with $\mathrm{MeOH}$ until complete removal of $\mathrm{Et}_{3} \mathrm{~N}$, and the residue was crystallized from 95\% ethanol to give $\mathbf{1 3}(0.32 \mathrm{~g}, 84 \%$ yield) as a pale yellow powder: m.p. $60-62{ }^{\circ} \mathrm{C}$; IR (KBr) $3420 \mathrm{~cm}^{-1}(\mathrm{OH}), 2222 \mathrm{~cm}^{-1}(\mathrm{CN})$ and $1644 \mathrm{~cm}^{-1}(\mathrm{C}=\mathrm{O}$, amide); ${ }^{1} \mathrm{H}-\mathrm{NMR}$ (DMSO- $\left.d_{6} / \mathrm{D}_{2} \mathrm{O}\right) \delta 1.55-1.86(10 \mathrm{H}, \mathrm{m}$, cyclohexyl), $2.26(1 \mathrm{H}, \mathrm{d}, \mathrm{H} 1$-cyclohexyl, $J=2.62 \mathrm{~Hz}), 3.31$ $\left(3 \mathrm{H}, \mathrm{s}, \mathrm{OC}_{3}\right), 3.47\left(2 \mathrm{H}, \mathrm{t}, \underline{\mathrm{C}}_{2 \mathrm{~d}}, J=5.60 \mathrm{~Hz}\right), 4.14(2 \mathrm{H}, \mathrm{t}$, $\left.\mathrm{C}_{2 \mathrm{c}}, J=6.52 \mathrm{~Hz}\right), 4.49\left(2 \mathrm{H}, \mathrm{s}, \mathrm{C}_{2 \mathrm{a}}\right), 7.03-7.34(5 \mathrm{H}, \mathrm{m}$, Ar-H); Anal. Calcd for $\mathrm{C}_{22} \mathrm{H}_{26} \mathrm{~N}_{2} \mathrm{O}_{4}$ (382.45): C, 69.09; H, 6.85; N, 7.32. Found: C, 69.06; H, 6.87; N, 7.32.

6-Cyclohexyl-2-((2-hydroxyethoxy)methoxy)-4-(4methoxyphenyl)-nicotinonitrile (15). A mixture of $\mathbf{1 4}$ $(0.42 \mathrm{~g}, 1.0 \mathrm{mmol}), \mathrm{Et}_{3} \mathrm{~N}(1 \mathrm{~mL})$, and $\mathrm{H}_{2} \mathrm{O}(0.1 \mathrm{~mL})$ in $\mathrm{MeOH}(5 \mathrm{~mL})$ was stirred overnight at room temperature. The volatiles were evaporated under reduced pressure. The residue was repeatedly co-evaporated with $\mathrm{MeOH}$ until complete removal of $\mathrm{Et}_{3} \mathrm{~N}$, and the residue was purified by a silica gel column chromatography (eluate: $6 \% \mathrm{MeOH}$ in $\left.\mathrm{CH}_{2} \mathrm{Cl}_{2}\right)$ to give 15 ( $0.33 \mathrm{~g}, 87 \%$ yield $)$ as a colorless syrup: IR (KBr) $3428 \mathrm{~cm}^{-1}(\mathrm{OH})$ and $2222 \mathrm{~cm}^{-1}(\mathrm{CN}) ;{ }^{1} \mathrm{H}-$ NMR (DMSO- $d_{6}$ ) $\delta 1.24-1.89$ (10H, m, cyclohexyl), 2.69 $(1 \mathrm{H}, \mathrm{t}$, cyclohexyl-H-1, $J=2.83 \mathrm{~Hz}), 3.45\left(2 \mathrm{H}, \mathrm{t}, \mathrm{C}_{2}(\mathrm{~d})\right.$, $J=5.6 \mathrm{~Hz}), 3.75\left(2 \mathrm{H}, \mathrm{t}, \mathrm{CH}_{2}(\mathrm{c}), J=4.43 \mathrm{~Hz}\right), 3.83(3 \mathrm{H}, \mathrm{s}$, $\left.\mathrm{OCH}_{3}\right), 4.45\left(2 \mathrm{H}, \mathrm{s}, \mathrm{CH}_{2}(\mathrm{a})\right), 4.87(1 \mathrm{H}, \mathrm{t}, \mathrm{OH}, J=4.65 \mathrm{~Hz}$, exchange with $\left.\mathrm{D}_{2} \mathrm{O}\right), 7.06(1 \mathrm{H}, \mathrm{s}, \mathrm{H} 5$-pyridine- $), 7.09(2 \mathrm{H}, \mathrm{d}$, $\mathrm{Ar}-\mathrm{H}, J=8.00 \mathrm{~Hz}), 7.61(2 \mathrm{H}, \mathrm{d}, \mathrm{Ar}-\mathrm{H}, J=7.69 \mathrm{~Hz})$; Anal. Calcd. for $\mathrm{C}_{22} \mathrm{H}_{26} \mathrm{~N}_{2} \mathrm{O}_{4}$ (382.45): C, 69.09; H, 6.85; N, 7.32. Found: C, 69.11; H, 6.88; N, 7.30.

6-Cyclohexyl-4-(4-methoxyphenyl)-2-oxo-1-(prop2-yn-1-yl)-1,2-dihydropyridine-3-carbonitrile (16) and 6-Cyclohexyl-4-(4-methoxyphenyl)-2-(prop-2-yn-1-yloxy) nicotinonitrile (17). A mixture of $\mathbf{3}(2 \mathrm{~g}, 6.48 \mathrm{mmol})$ with propargyl bromide $(0.7 \mathrm{~g}, 6.5 \mathrm{mmol})$ was added to dry acetone $(20 \mathrm{~mL})$ in the presence anhydrous $\mathrm{K}_{2} \mathrm{CO}_{3}(0.9 \mathrm{~g}$, $6.5 \mathrm{mmol}$ ); the reaction mixture was heated under reflux for $15 \mathrm{~h}$. The inorganic residue was removed by filtration; the solvent was evaporated under reduced pressure and then the formed solid product was separated by silica gel column chromatography (eluate: $\mathrm{CH}_{2} \mathrm{Cl}_{2}$ to $1 \% \mathrm{MeOH} /$ $\left.\mathrm{CH}_{2} \mathrm{Cl}_{2}\right)$ to give $\mathbf{1 6}(0.67 \mathrm{~g}, 30 \%$ yield $)$ as a yellow oil and 17 (0.65 g, 29\% yield) as pale yellow oil. Spectral and analytical data for 16: $\mathrm{IR}(\mathrm{KBr}) 2216 \mathrm{~cm}^{-1}(\mathrm{CN})$ and $1641 \mathrm{~cm}^{-1}$ $\left(\mathrm{C}=\mathrm{O}\right.$, amide); ${ }^{1} \mathrm{H}-\mathrm{NMR}$ (DMSO- $\left.d_{6}\right) \delta 1.14-1.96(10 \mathrm{H}$, $\mathrm{m}$, cyclohexyl), $2.90(1 \mathrm{H}, \mathrm{t}, \mathrm{H}-1$-cyclohexyl, $J=2.81 \mathrm{~Hz})$, $3.34(1 \mathrm{H}, \mathrm{s}, \equiv \mathrm{C}-\underline{\mathrm{H}}), 3.84\left(3 \mathrm{H}, \mathrm{s}, \mathrm{OC}_{3}\right), 4.99(2 \mathrm{H}, \mathrm{d}, N-$ $\left.\mathrm{CH}_{2}, J=2.41 \mathrm{~Hz}\right), 6.46(1 \mathrm{H}, \mathrm{s}, \mathrm{H}-5$-pyridone $), 7.09(2 \mathrm{H}$, $\mathrm{d}, \mathrm{Ar}-\mathrm{H}, J=8.69 \mathrm{~Hz}), 7.65(2 \mathrm{H}, \mathrm{d}, \mathrm{Ar}-\mathrm{H}, J=9.01 \mathrm{~Hz})$; Anal. Calcd for $\mathrm{C}_{22} \mathrm{H}_{22} \mathrm{~N}_{2} \mathrm{O}_{2}$ (346.42): C, 76.28; H, 6.40; N, 8.09. Found: C, 76.26; H, 6.44; N, 8.08. Spectral and analytical data for 17: $\mathrm{IR}(\mathrm{KBr}) 2222 \mathrm{~cm}^{-1}(\mathrm{CN}) ;{ }^{1} \mathrm{H}-\mathrm{NMR}$ (DMSO- $\left.d_{6}\right) \delta 1.22-1.93(10 \mathrm{H}, \mathrm{m}$, cyclohexyl), $2.72(1 \mathrm{H}, \mathrm{t}$, $\mathrm{H}-1$-cyclohexyl, $J=2.78 \mathrm{~Hz}), 3.51(1 \mathrm{H}, \mathrm{s}, \equiv \mathrm{C}-\underline{\mathrm{H}}), 3.84(3 \mathrm{H}$, $\left.\mathrm{s}, \mathrm{OC}_{3}\right), 5.14\left(2 \mathrm{H}, \mathrm{d}, \mathrm{OC}_{2}, J=2.42 \mathrm{~Hz}\right), 7.09-7.12(3 \mathrm{H}$, $\mathrm{m}, \mathrm{Ar}-\mathrm{H}$ and $\mathrm{H} 5$-pyridone), $7.61(2 \mathrm{H}, \mathrm{d}, \mathrm{Ar}-\mathrm{H}, J=8.69 \mathrm{~Hz})$; Anal. Calcd for $\mathrm{C}_{22} \mathrm{H}_{22} \mathrm{~N}_{2} \mathrm{O}_{2}$ (346.42): C, 76.28; $\mathrm{H}, 6.40 ; \mathrm{N}$, 8.09. Found: C, 76.25; H, 6.45; N, 8.06.

1-Allyl-6-cyclohexyl-4-(4-methoxyphenyl)2-oxo-1,2-dihydropyridine-3-carbonitrile (18) and 2-(Allyloxy)-6-cyclohexyl-4-(4-methoxyphenyl)nicotinonitrile (19): A mixture of $\mathbf{3}$ (1 g, $3.24 \mathrm{mmol})$ with allyl bromide $(0.36 \mathrm{~g}, 3.0 \mathrm{mmol})$ was added to dry acetone $(10 \mathrm{~mL})$ in the presence anhydrous $\mathrm{K}_{2} \mathrm{CO}_{3}(0.45 \mathrm{~g}, 3.27 \mathrm{mmol})$; the reaction mixture was heated under reflux for $15 \mathrm{~h}$. The inorganic residue was removed by filtration; the solvent was evaporated under reduced pressure and then the formed solid product was separated by silica gel column chromatography (eluate: $\mathrm{CH}_{2} \mathrm{Cl}_{2}$ to $\left.5 \% \mathrm{EtOAc} / \mathrm{CH}_{2} \mathrm{Cl}_{2}\right)$ to give $\mathbf{1 8}(0.37 \mathrm{~g}$, $32 \%$ yield) as a yellow powder and $\mathbf{1 9}(0.39 \mathrm{~g}, 39 \%$ yield $)$ as a white powder. Spectral and analytical data for 19: m.p. 90-92 ${ }^{\circ} \mathrm{C}$; IR (KBr) $2213 \mathrm{~cm}^{-1}(\mathrm{CN})$ and $1644 \mathrm{~cm}^{-1}$ $(\mathrm{C}=\mathrm{O}$, amide $) ;{ }^{1} \mathrm{H}-\mathrm{NMR}\left(\mathrm{DMSO}_{6} \mathrm{~d}_{6}\right) \delta 1.22-1.91(10 \mathrm{H}$, $\mathrm{m}$, cyclohexyl), $2.70(1 \mathrm{H}, \mathrm{t}$, cyclohexyl-H-1, $J=2.39 \mathrm{~Hz})$, $2.84\left(3 \mathrm{H}, \mathrm{s}, \mathrm{OCH}_{3}\right), 4.98\left(2 \mathrm{H}, \mathrm{d}, \mathrm{CH}_{2}(\mathrm{a}), J=4.49 \mathrm{~Hz}\right), 5.31$ $(2 \mathrm{H}, \mathrm{d}, \mathrm{Hc}, J=10.49 \mathrm{~Hz}), 5.43(2 \mathrm{H}, \mathrm{d}, \mathrm{Hc}, J=17.41 \mathrm{~Hz})$, $6.12(1 \mathrm{H}, \mathrm{m}, \mathrm{Hb}), 7.06(1 \mathrm{H}, \mathrm{s}$, pyridone-H-5), $7.09(2 \mathrm{H}, \mathrm{d}$, Ar-H, $J=8.69), 7.60$ (2H, d, Ar-H, $J=8.69)$; Anal. Calcd for $\mathrm{C}_{22} \mathrm{H}_{24} \mathrm{~N}_{2} \mathrm{O}_{2}$ (348.44): C, 75.83; H, 6.94; N, 8.04. Found: C, 75.85; H, 6.95; N, 8.03. Spectral and analytical data for 18: m.p. $60-62{ }^{\circ} \mathrm{C}$; IR (KBr) $2220 \mathrm{~cm}^{-1}(\mathrm{CN}) ;{ }^{1} \mathrm{H}-\mathrm{NMR}$ $\left(\right.$ DMSO- $\left.d_{6}\right) \delta 1.15-1.86(10 \mathrm{H}, \mathrm{m}$, cyclohexyl), $2.72(1 \mathrm{H}$, t, cyclohexyl-H-1, $J=2.49 \mathrm{~Hz}), 3.85\left(3 \mathrm{H}, \mathrm{s}, \mathrm{OCH}_{3}\right), 4.79$ $\left(2 \mathrm{H}, \mathrm{d}, \mathrm{CH}_{2}(\mathrm{a}), J=4.49 \mathrm{~Hz}\right), 5.06(1 \mathrm{H}, \mathrm{d}, \mathrm{Hc}, J=17.40 \mathrm{~Hz})$, $5.19(1 \mathrm{H}, \mathrm{d}, \mathrm{Hc}, J=10.79 \mathrm{~Hz}), 5.92-6.05(1 \mathrm{H}, \mathrm{m}, \mathrm{Hb}), 6.42$ (1H, s, pyridone-H-5), 7.09 (2H, d, Ar-H, $J=8.69 \mathrm{~Hz}), 7.64$ $\left(2 \mathrm{H}, \mathrm{d}, \mathrm{Ar}-\mathrm{H}, J=9.01 \mathrm{~Hz}\right.$ ); Anal. Calcd for $\mathrm{C}_{22} \mathrm{H}_{24} \mathrm{~N}_{2} \mathrm{O}_{2}$ 
(348.44): C, 75.83; H, 6.94; N, 8.04. Found: C, 75.80; H, $6.92 ; \mathrm{N}, 8.07$.

6-Cyclohexyl-4-(4-methoxyphenyl)-1-(oxiran2-ylmethyl)-2-oxo-1,2-dihydropyridine-3-carbonitrile (20). A mixture of $3(1 \mathrm{~g}, 3.24 \mathrm{mmol})$ and anhydrous $\mathrm{K}_{2} \mathrm{CO}_{3}(0.45 \mathrm{~g}, 3.27 \mathrm{mmol})$ in dry acetone $(10 \mathrm{~mL})$ was added to epichlorohydrin $(0.27 \mathrm{~g}, 3.0 \mathrm{mmol})$, and the mixture was heated under reflux for $15 \mathrm{~h}$. The inorganic residue was removed by filtration; the solvent was evaporated under reduced pressure and then the formed solid product was purified by silica gel column chromatography (eluate: $1 \% \mathrm{MeOH}$ in $\left.\mathrm{CH}_{2} \mathrm{Cl}_{2}\right)$ to give $20(0.47 \mathrm{~g}, 42 \%)$ as a colorless syrup, IR (KBr) $2222 \mathrm{~cm}^{-1}(\mathrm{CN})$ and $1608 \mathrm{~cm}^{-1}$ $\left(\mathrm{C}=\mathrm{O}\right.$, amide); ${ }^{1} \mathrm{H}-\mathrm{NMR}$ (DMSO- $\left.d_{6}\right) \delta 1.14-1.84(10 \mathrm{H}, \mathrm{m}$, cyclohexyl), $2.51(1 \mathrm{H}, \mathrm{t}$, cyclohexyl, $J=2.81 \mathrm{~Hz}), 3.83(3 \mathrm{H}$, $\left.\mathrm{s}, \mathrm{OCH}_{3}\right), 4.33-4.36(1 \mathrm{H}, \mathrm{m}, \mathrm{Hc}$-oxiran ring $), 4.44(1 \mathrm{H}, \mathrm{t}$, Hc-oxiran ring, $J=4.82 \mathrm{~Hz}), 4.49-4.60(2 \mathrm{H}, \mathrm{m}, \mathrm{Ha}$, and $\mathrm{Hb}), 5.44(1 \mathrm{H}, \mathrm{m}, \mathrm{Ha}$ '), 7.04 (1H, s, H5-pyridone), $7.11(2 \mathrm{H}$, d, Ar-H, $J=9.29 \mathrm{~Hz}), 7.57(2 \mathrm{H}, \mathrm{d}, \mathrm{Ar}-\mathrm{H}, 8.69 \mathrm{~Hz}$ ); Anal. Calcd for $\mathrm{C}_{22} \mathrm{H}_{24} \mathrm{~N}_{2} \mathrm{O}_{3}$ (364.44): C, 72.50; $\mathrm{H}, 6.64 ; \mathrm{N}, 7.69$. Found: C, 72.55; H, 6.66; N, 7.68.

\section{References}

1. R. Leclercq, P. Courvalin, Clin. Infect. Dis. 24, 545 (1997)

2. C. J. Soussy, J. Nguyen, F. Goldstein, H. Dabernat, A. Andremont, R. Leclercq, H. Drugeon, P. Cavallo, H. Chardon, J. Etienne, Y. Rio, P. Courvalin, Clinical microbiology and infection: the official publication of the european society of clinical microbiology and infectious diseases9 (2003) 997

3. https://www.cdc.gov/drugresistance/pdf/ar-threats-2013-508

4. V.V. Gostev, O.S. Kalinogorskaya, L.N. Popenko, T.V. Chernenkaya, Z.S. Naumenko, T.M. Voroshilova, Y.A. Zakharova, O.E. Khokhlova, A.N. Kruglov, M.G. Ershova IV, S.V.Sidorenko Molchanova, Antibiot. Khimioter. 60, 3 (2015)

5. A. Kali, S. Stephen, S. Umadevi, S. Kumar, N.M. Joseph, S. Srirangaraj, J. Clin. Diagn. Res. 7, 1979 (2013)

6. K. Kikuchi, Gekkan Yakuji 48, 1489 (2006)

7. H. Komatsuzawa, M. Sugai, Igaku no Ayumi 221, 469 (2007)

8. D.M. Livermore, A. Pearson, Clin. Microbiol. Infect. 13, 7 (2007)

9. Y-p Wang, Shanxi Yiyao Zazhi 42, 460 (2013)

10. L. Zhang, X-d Tong, Sichuan Yixue 34, 1030 (2013)

11. A. Corso, L. Guerriero, F. Pasteran, P. Ceriana, R. Callejo, M. Prieto, E. Tuduri, H. Lopardo, C. Vay, J. Smayevsky, M. Tokumoto, J.M. Alvarez, P.R. Pardo, M. Galas, Rev. Panam. Salud Publica 30, 619 (2011)

12. R.F. Pfeltz, B.J. Wilkinson, Curr. Drug Targets Infect. Disord. 4, 273 (2004)

13. M.M.M. Bahgat, A.A. Elbialy, M.M.M. Zaky, S.M.E. Toubar, Int. J. Curr. Microbiol. Appl. Sci. 4, 460 (2015)

14. A. Breves, C.A.C. Miranda, C. Flores, I. De Filippis, M.M. Clementino, J. Bras. Patol. Med. Lab. 51, 143 (2015)

15. T.E.-S. El-Banna, F.I. Sonbol, A.A. Abd El-Aziz, E.A.-S. ElEkhnawy, Int. J. Curr. Microbiol. Appl. Sci. 4, 1 (2015)

16. M.M. Osman, M.M. Osman, N.A. Mohamed, S.M. Osman, M. Magzoub, S.M. El-Sanousi, Am. J. Microbiol. Res. 4, 56 (2016)

17. D. Chudobova, S. Dostalova, I. Blazkova, P. Michalek, B. RuttkayNedecky, M. Sklenar, L. Nejdl, J. Kudr, J. Gumulec, K. Tmejova, M. Konecna, M. Vaculovicova, D. Hynek, M. Masarik, J.
Kynicky, R. Kizek, V. Adam, Int. J. Environ. Res. Public Health 11, 3233 (2014)

18. R.S. Edlin, D.J. Shapiro, A.L. Hersh, H.L. Copp, J. Urol. 190, 222 (2013)

19. K. Lee, M.N. Kim, J.S. Kim, H.L. Hong, J.O. Kang, J.H. Shin, Y.J. Park, D. Yong, S.H. Jeong, Y. Chong, Yonsei Med. J. 52, 793 (2011)

20. F.A. Rosenberg, H.H. Duquino, Toxic. Assess. 4, 281 (1989)

21. I.K. Voukeng, V.P. Beng, V. Kuete, BMC Complement. Altern. Med. 16, 388 (2016)

22. S. Zhang, Y. Zhang, M. Piao, Y. Wang, Yanbian Daxue Yixue Xuebao 23, 285 (2000)

23. O. Adenaike, O.S. Olonitola, J.B. Ameh, C.M.Z. Whong, Int. J. Curr. Microbiol. Appl. Sci. 5, 796 (2016)

24. J.V. McArthur, D.E. Fletcher, R.C. Tuckfield, C. Baker-Austin, Microb. Ecol. 72, 840 (2016)

25. G. Moka, H. Shehu, F. Voci, L. Turmalaj, R. Bajramaj, J. Anim. Vet. Adv. 11, 4571 (2012)

26. Y. Xue, J. Chen, Y. Hua, W. Zhang, S. Liu, D. Liu, Pak. J. Zool. 48, 1155 (2016)

27. H.J. Jessen, K. Gademann, Nat. Prod. Rep. 27, 1168 (2010)

28. S.R. Vegi, S.K. Boovanahalli, B. Patro, K. Mukkanti, Eur. J. Med. Chem. 46, 1803 (2011)

29. M.E. Goldman, J.H. Nunberg, J.A. O'Brien, J.C. Quintero, W.A. Schleif, K.F. Freund, S.L. Gaul, W.S. Saari, J.S. Wai, J.M. Hoffman, Proc. Natl. Acad. Sci. 88, 6863 (1991)

30. R. Gomez, S. Jolly, T. Williams, T. Tucker, R. Tynebor, J. Vacca, G. McGaughey, M.-T. Lai, P. Felock, V. Munshi, D. DeStefano, S. Touch, M. Miller, Y. Yan, R. Sanchez, Y. Liang, B. Paton, B.-L. Wan, N. Anthony, Bioorganic Med. Chem. Lett. 21, 7344 (2011)

31. G.N. Anilkumar, C.A. Lesburg, O. Selyutin, S.B. Rosenblum, Q. Zeng, Y. Jiang, T.-Y. Chan, H. Pu, H. Vaccaro, L. Wang, F. Bennett, K.X. Chen, J. Duca, S. Gavalas, Y. Huang, P. Pinto, M. Sannigrahi, F. Velazquez, S. Venkatraman, B. Vibulbhan, S. Agrawal, N. Butkiewicz, B. Feld, E. Ferrari, Z. He, C.-K. Jiang, R.E. Palermo, P. McMonagle, H.C. Huang, N.-Y. Shih, G. Njoroge, J.A. Kozlowski, Bioorg. Med. Chem. Lett. 21, 5336 (2011)

32. A. Batra, S. Nandi, M.C. Bagchi, Med. Chem. Res. 24, 2432 (2015)

33. K.X. Chen, C.A. Lesburg, B. Vibulbhan, W. Yang, T.Y. Chan, S. Venkatraman, F. Velazquez, Q. Zeng, F. Bennett, G.N. Anilkumar, J. Duca, Y. Jiang, P. Pinto, L. Wang, Y. Huang, O. Selyutin, S. Gavalas, H. Pu, S. Agrawal, B. Feld, H.-C. Huang, C. Li, K.-C. Cheng, N.-Y. Shih, J.A. Kozlowski, S.B. Rosenblum, F.G. Njoroge, J. Med. Chem. 55, 2089 (2012)

34. N. Singh, B. Shaik, N. Agrawal, K. Anita, V.K. Agrawal, S.P. Gupta, Lett. Drug Des. Discov. 13, 757 (2016)

35. Z. Lv, C. Sheng, T. Wang, Y. Zhang, J. Liu, J. Feng, H. Sun, H. Zhong, C. Niu, K. Li, J. Med. Chem. 53, 660 (2010)

36. A. Scala, M. Cordaro, F. Risitano, I. Colao, A. Venuti, M.T. Sciortino, P. Primerano, G. Grassi, Mol. Divers. 16, 325 (2012)

37. Q. Li, L.A. Mitscher, L.L. Shen, Med. Res. Rev. 20, 231 (2000)

38. D.T.W. Chu, Med. Res. Rev. 19, 497 (1999)

39. L. Jayasinghe, H.K. Abbas, M.R. Jacob, W.H.M.W. Herath, N.P.D. Nanayakkara, J. Nat. Prod. 69, 439 (2006)

40. X. Fan, D. Feng, Y. Qu, X. Zhang, J. Wang, P.M. Loiseau, G. Andrei, R. Snoeck, E.D. Clercq, Bioorganic Med. Chem. Lett. 20, 809 (2010)

41. M. Kumarihamy, F.R. Fronczek, D. Ferreira, M. Jacob, S.I. Khan, N.P.D. Nanayakkara, J. Nat. Prod. 73, 1250 (2010)

42. R.A.I. Abou-Elkhair, A.H. Moustafa, A.Z. Haikal, A.M. Ibraheem, Eur. J. Med. Chem. 74, 388 (2014)

43. S.K. Sunil, K. Rai, R.S. Khanna, A.K. Tewari, RSC Adv. 4, 44141 (2014) 
44. S. Balalaie, M.M. Hashemi, S.H. Khezri, F. Rominger, E. Ghabraie, T. Oeser, J. Heterocycl. Chem. 50, 1272 (2013)

45. A. Maleki, Tetrahedron Lett. 16, 2055 (2013)

46. A. Maleki, RSC Adv. 4(109), 64169 (2014)

47. A.G. Maleki, M.R. Firouzi-Haji, Green. Pure Appl. Chem. 90, 387 (2018)

48. A.M. Maleki, H.R. Paydar, RSC Advances. 6, 13657 (2016)

49. P.A. Abolghasem Davoodnia, A. Morsali, H. Eshghi, N. TavakoliHoseini, S. Khadem, Bull. Korean Chem. Soc. 32, 1873 (2011)

50. A.A.P. Davoodnia, A. Morsali, H. Eshghi, N. Tavakoli-Hoseini, S. Khadem, Bull. Korean Chem. Soc. 23, 1873 (2011)

51. M.G.M.D. Al-Saad, P. Quadrelli, ChemistrySelect 2, 10340 (2017)

52. A. Kamal, V. Tekumalla, A. Krishnan, M. Pal-Bhadra, U. Bhadra, ChemMedChem 3, 794 (2008)
53. T. Legigan, J. Clarhaut, I. Tranoy-Opalinski, A. Monvoisin, B. Renoux, M. Thomas, A. LePape, S. Lerondel, S. Papot, Angew. Chem. 51, 11606 (2012)

54. M. Wawryszyn, P.F. Sauter, M. Nieger, M.R.M. Koos, C. Koehler, B. Luy, E.A. Lemke, S. Braese, Eur. J. Org. Chem. 2018, 4296 (2018)

55. K.M. Craft, S.D. Townsend, Carbohydr. Res. 440-441, 43 (2017)

56. J. Krim, M. Taourirte, J.W. Engels, Molecules 17, 179 (2012)

57. S. Broussy, Y. Coppel, M. Nguyen, J. Bernadou, B. Meunier, Chem. Eur. J. 9, 2034 (2003)

58. S. Vertuani, A. Baldisserotto, K. Varani, P.A. Borea, B.M.M. De Cruz, L. Ferraro, S. Manfredini, A. Dalpiaz, Eur. J. Med. Chem. 54, $202(2012)$ 